\title{
LA ALTERNANCIA DE LAS PREPOSICIONES POR Y DE COMO INTRODUCTORAS DEL COMPLEMENTO AGENTE
}

\author{
María Soledad Funes \\ Universidad de Buenos Aires - Conicet \\ solefunes@gmail.com
}

\begin{abstract}
Resumen
En el marco teórico del Enfoque Cognitivo-Prototípico (ECP), el presente trabajo describe comparativamente el uso en contextos auténticos de construcciones pasivas (CP) con Complemento Agente (CAg) introducidos por por y por de. Se espera aportar evidencia empírica a la siguiente hipótesis: el CAg introducido por la preposición por tiende a aparecer en contextos con pacientes altamente afectados y con agentes [+humanos] y [+determinados], en esquemas de predicaciones de eventos dinámicos; mientras que el agente introducido por de suele tener los atributos [-humano] y [-determinado], en contextos de pacientes con baja afectación, y en esquemas de predicaciones con entidades estáticas.
\end{abstract}

PALABRAS CLAVE: voz pasiva, 'complemento agente', preposición por, preposición de, Enfoque Cognitivo-Prototípico (ECP).

\begin{abstract}
Within the framework of PrototypicalCognitive Approach, the present study describes comparatively the use of passive constructions (PC) with Agent Complement (AgC) introduced by por and de in authentic contexts. It's expected to provide empirical evidence to the following hypothesis: the AgC introduced by the preposition por tends to appear in contexts with heavily affected patients and [+human] and [+specific] agents, in schemes of dynamic events, while the $\mathrm{AgC}$ introduced by de tends to have the attributes [-human] and [-specific] in context of low affected patients and schemes of static entities' predications.
\end{abstract}

KEY WORDS: passive voice, 'agent complement', preposition por, preposition de, Prototypical-Cogntive Approach.

\section{Introducción}

El presente trabajo describe comparativamente el uso en contextos auténticos de construcciones pasivas (CP) con Complementos Agente (CAg) introducidos por la preposición por y por la preposición de, con el objetivo de ofrecer una caracterización sistemática de los actantes de ambas construcciones. Se parte del presupuesto teórico del Enfoque Cognitivo Prototípico (ECP) de que la Sintaxis está motivada por la Semántica y la Pragmática, es decir, todo uso de una forma es significativo y responde a una organización sistemá- 
tica (Langacker, 1991; Lakoff, 1987, entre otros). Desde el ECP se sostiene la no variación libre entre formas distintas. El valor de uso de las formas se explica a partir de sus contextos de aparición, medidos en forma cualitativa y cuantitativa. Asimismo, se consideran los resultados de un trabajo previo (Funes, en prensa), donde se caracterizó el CAg introducido por la preposición de en su contexto preferido de aparición, comparándolo con los atributos del agente y del paciente de la cláusula transitiva prototípica (Hopper y Thompson, 1980). En ese trabajo, se concluyó que: (i) en corpus oral hay un uso bastante marginal del CAg introducido por la preposición de; (ii) cuando el hablante elige la preposición de para introducir un CAg, lo hace para acompañar agentes con los atributos [-humano] y [-determinado], (iii) en contextos de sujetos pacientes con baja afectación, y en esquemas de predicaciones con entidades estáticas preexistentes.

Siguiendo esta línea de análisis, el presente estudio proporciona evidencia empírica a la siguiente hipótesis de trabajo propuesta para los corpora orales: en contraste con el CAg introducido por de, el CAg introducido por la preposición por tiende a aparecer en contextos con sujetos pacientes altamente afectados y con agentes [+humanos] y [+determinados], en esquemas de predicaciones de eventos dinámicos. Asimismo, se aporta evidencia empírica a las hipótesis propuestas (tanto en el estudio previo acerca del CAg introducido por de como en el presente trabajo), mediante el análisis de un corpus escrito.

En función de las exigencias del marco teórico, se analizan cuantitativa y cualitativamente ejemplos provenientes de corpora orales y un corpus escrito de la Ciudad de Buenos Aires. Se estudian los siguientes atributos de los actantes de la CP: [+/- humano], [+/- determinado] y el grado de afectación del sujeto paciente.

Cabe aclarar que se relevó la presencia de CAg introducido por la preposición por y por de en contextos de sentido pasivo, tanto de voz pasiva de frase verbal con ser como de construcción pronominal con se, en contextos de 'estar + atributoll $^{\text {y en predicaciones no finitas }}{ }^{2}$.

1 Con respecto a la existencia de sentido pasivo en las construcciones con verbo 'estar', véanse, entre otros: Esbozo (1973: §3.12.8.), Rosetti (1969a: §5.3.), Seco (1999: §8.8.5.), Delbecque (2003: 6 y ss.) y GRAE (2009: §28.16d y e).

2 En el presente trabajo, se denomina 'predicación no finita' a aquélla que no tiene verbo, pero que es tratada como predicación tanto por la semántica eventiva de la base del participio como por asociar dos actantes con roles claros (en términos de la cláusula transitiva prototípica) y en el caso de las CP tratadas aquí, explícitos (agente y paciente presentes en la cláusula). Al respecto de este tipo de construcciones, la GRAE de 2009 reconoce el sentido pasivo de oraciones como Una novela leída por todo el mundo, a las que llama "oraciones pasivas sin verbo ser, y aclara que para algunas gramáticas estas estructuras se denominan "relativas reducidas" (2009: §41.2b). 


\section{Breve presentación del marco teórico: el Enfoque Cognitivo-Prototípico}

El Enfoque Cognitivo-Prototípico parte de la idea de que la organización lingüística está caracterizada en términos del procesamiento cognitivo de los seres humanos (Langacker, 1987: 1). Se trata, por lo tanto, de un intento de describir y explicar la lengua siguiendo los procesos mentales que usan los seres humanos para dar razón y sentido al mundo que nos rodea.

Siguiendo este punto de partida, desde el ECP se sostiene que: i) la estructura semántica no es universal, sino propia de las diferentes lenguas; ii) la gramática (sintaxis) no constituye un nivel formal de representación autónomo. En cambio, se encuentra motivada por la semántica y la pragmática; iii) de esto último se sigue que no existe la separación tradicional en módulos propuesta por el Generativismo (Fonología, Morfología, Sintaxis y Léxico). Estos niveles, en realidad, forman un continuum de estructuras significativas permanentemente interrelacionado. Pueden ser divididos en componentes separados sólo de manera arbitraria. Además, el lenguaje no se puede separar tajantemente de otras facultades de la cognición humana, por lo que la intención comunicativa y el punto de vista del hablante resultan fundamentales dentro de la metodología de este enfoque.

Como consecuencia de esta concepción, el ECP sostiene que el signo en su constitución está motivado por el objetivo comunicativo, de ahí que se hable de una Gramática Emergente del discurso (Hopper, 1988). Desde esta perspectiva, las estructuras o regularidades provienen del discurso y toman forma a partir de él en un proceso permanente de construcción de la Gramática. El término emergente se refiere a la naturaleza esencialmente provisional y lábil de la relación entre forma y significado. Los signos (tanto en su forma como en su función) están sujetos a los actos de comunicación; esto significa que tanto la forma como la función del signo son provisionales y dependen no de un núcleo semántico inherente y constante sino de la comunicación. La gramática de una lengua consiste, por lo tanto, no en un sistema uniforme, simple y delimitado sino en una colección abierta de formas que están siendo constantemente reestructuradas y resemantizadas en el uso. La gramática, entonces, es un conjunto de convenciones sedimentadas que se han rutinizado a partir de la frecuencia ${ }^{3}$.

3 En palabras de Hopper: "The notion of Emergent Grammar is meant to suggest that structure, or regularity, comes out of discourse and is shaped by discourse in an ongoing process. Grammar is, in this view, simply the name for certain categories of observed repetitions in discourse." (1988: 156) "The gramar of a language, then, consists not of a single delimited system, but rather, of an open-ended collection of forms that are constantly being restructured and resemanticized during actual use" (1988: 159). 
En este sentido, el hecho de que un hablante elija usar la preposición por o la preposición de para encabezar un CAg se relaciona con el objetivo comunicativo que persigue y con un significado específico que quiere transmitir.

\section{Estado de la cuestión}

\subsection{La voz pasiva: algunas caracterizaciones}

Las gramáticas hispánicas oracionales han estudiado el fenómeno de la voz pasiva desde distintos enfoques: la Gramática de la Real Academia Española (GRAE) de 1931 (§83) y Alonso/H. Ureña (1938-1939: §128) estudian la voz pasiva desde un enfoque morfológico, es decir, analizan el cambio de voz en español como un accidente o morfema verbal que señala si la significación del verbo es producida o recibida por el sujeto. Bello (1980[1847]: §430), Alcina Franch/Blecua (1975: \$5.4.1.), Rosetti (Rosetti, 1969a y b: 72), Kovacci (1990-1992: 69), la Gramática Descriptiva de la Lengua Española (GDLE, 1999) y la GRAE de 20095 entre otros, analizan la CP desde un enfoque sintáctico, esto es, describen las oraciones pasivas como construcciones marcadas, donde el verbo toma una forma particular y el paciente toma el lugar del agente, que se transforma en término oblicuo o desaparece. Por su parte, Lenz (1935: $\$ 57$ y §253), Gili Gaya (1955: §56), el Esbozo (1973:\$3.5.2/3) y Seco (1999: 187) analizan la CP desde un enfoque semántico-psicológico, según el cual, el hablante tiene interés por el objeto/paciente. Alarcos Llorach (1966: §3), en contraposición con el resto de las Gramáticas, considera que no existe la voz pasiva en español. En este sentido, ejemplos como Juan fue vencido y Juan fue vencedor son estructuras atributivas. Para este autor, el sentido pasivo resulta sólo del contenido total de la oración sin presentar manifestación morfológica y/o sintáctica de ningún tipo.

4 Dentro de la GDLE, se destaca el artículo de Mendikoetxea, para quien las construcciones pasivas (derivadas de la activa) están dentro de la clase general de las construcciones inacusativas. La autora también reconoce construcciones pasivas con estar y otros verbos, y con formas no personales (sin auxiliar). En cuanto al significado de la CP, Mendikoetxea señala que al ser el objeto nocional el punto de partida de la construcción, el objeto cumple las funciones discursivas de tema y tópico. Luego, añade: "Las oraciones de pasiva perifrástica implican siempre la existencia de un agente o causa que pueden quedar implícitos o pueden ser expresados por medio de un sintagma preposicional con por" (1999: §25.1.3). 5 La GRAE de 2009 señala que las oraciones pasivas son estructuras intransitivas que se forman con verbos transitivos. El paciente de la acción expresada que corresponde al complemento directo pasa a ser sujeto de la oración pasiva (\$41.2a). Es decir, estudian las $\mathrm{CP}$ desde un punto de vista sintáctico y las ven como derivadas de la voz activa. Asimismo, la GRAE de 2009 advierte que las oraciones pasivas expresan el mismo contenido que las activas correspondientes (es decir que tienen el mismo significado), pero que se prefieren las oraciones pasivas a las activas "cuando se desea destacar al paciente, cuando no se posee información sobre el agente o cuando se desea mantenerlo en segundo plano" (\$41.2f). 
Fuera del ámbito hispánico, a grandes rasgos, se han ofrecido tres propuestas para explicar la CP: una propuesta formal, proveniente de la teoría generativa, que consiste en definir la CP como la promoción del objeto y la consecuente 'democión' (degradación o descenso) del sujeto a oblicuo ${ }^{6}$; una propuesta funcionalista, ejemplificada en el planteo de Givón (1979:186), quien define la CP como una construcción sintáctica que topicaliza el 'paciente $^{7}$; y, por último, dos propuestas desde el Enfoque Cognitivo-prototípico: la hipótesis de Shibatani (1985) y el planteo de Borzi (2000, 2004, 2005, 2006).

Shibatani propone que la CP tiene como función principal la defocalización del agente (es decir, no puede explicarse como una transformación ni como una topicalización). Para esta autora, la estrategia de defocalización del agente incluiría los siguientes fenómenos: i) no mención del agente; ii) mención del agente en un espacio (slot) sintáctico no prominente; iii) borramiento de la identidad del agente a través del uso de formas plurales; y iv) referencia indirecta al agente a través del uso del caso oblicuo (1985: 832). Esta hipótesis puede explicar la existencia de voz pasiva de verbos intransitivos (como ocurre en lenguas como el latín y el galés), cuestión que no podían explicar las otras perspectivas.

\subsubsection{La propuesta de Borzi: un estudio desde el ECP para el español de Buenos Aires}

Desde el ECP, Borzi niega la derivación ${ }^{8}$ (como Shibatani) y analiza la CP como una construcción diferente con significado propio. Por un lado, en cuanto a la voz pasiva de frase verbal con ser, postula:

6 Véase, por ejemplo, Brucart (1990), quien, partiendo del modelo de rección y ligamiento, caracteriza la pasiva como "un procedimiento sintáctico que permite colocar como sujeto de un verbo agentivo el argumento que desempeña el papel temático de tema o paciente" (1990: 195). Al no otorgar caso ni papel temático el participio a su sujeto, el objeto directo ocupa la posición del sujeto y el agente se inhibe.

7 En palabras de Givón: "Passivization is the process by which a non-agent is promoted into the role of a main topic of the sentence. And to the extent that the language possesses coding properties which identify main topics as subjects and distinguish them from topics, then this promotion may also involve subjectivization" (1979: 186).

8 Borzi enuncia los siguientes argumentos que permiten sostener que la $\mathrm{CP}$ no es una derivación de la construcción activa: "1. Entre activa y pasiva no se mantiene la correspondencia caso/función (Agente/Sujeto-Paciente/Objeto); 2. Las funciones de la activa son distintas de las de pasiva (en una no puede aparecer nunca un complemento agente y en la otra no puede aparecer nunca un objeto directo); 3. En la voz pasiva aparece una frase verbal con una forma verbal nueva (no presente en la activa) y una desinencia en ado/ada(s) que concuerda en género y número con el sujeto paciente; 4 . El complemento agente no puede ser interpretado como un circunstancial más porque el complemento agente es diferente del complemento de causa (La noticia fue divulgada por los periódicos y por la radio y * por su interés. L. Carreter, 1975); 5 . El reemplazo por la forma lo predicativa no es paráfrasis 
[...] partiendo de los dos modelos cognitivos idealizados de cadena de acción o bola de billar y escenario (cfr. Langacker, 1991: cap. 7), la formulación pasiva se corresponde con una huella cognitiva de un hecho que es una desviación de estos modelos y ocupa una zona intermedia, compartiendo características con ambos. Es así como la formulación pasiva no pone en la mira la transferencia de energía (como típicamente ocurre en el modelo de cadena de acción), sino el desarrollo del cambio en el Paciente, o sea, cómo opera en el lugar del cambio la energía transmitida (la pasiva de ser no hace hincapié ni en el traspaso, ni en el resultado, sino en el proceso del cambio). El Paciente (Tema o lugar del cambio) goza así (como en el modelo de escenario) de alta autonomía conceptual. Equilibrando esto, el Agente por su lado no está en la mira pero sigue vigente. En este marco se considera además que las nociones de individualización o determinación del Paciente, Paciente conocido y Paciente tópico son tres formas de decir lo mismo. El hablante pone en la mira al Paciente porque lo conoce (2004: 4-5) .

Borzi ofrece esta descripción en términos de modelos cognitivos idealizados porque, desde el punto de vista situacional, no se describe la transferencia de la energía sino el desarrollo del cambio en el paciente, o sea, se describe cómo opera en el paciente la energía transmitida.

Por otro lado, en español también existe la pasiva con el pronombre se. En cuanto a esta construcción, Borzi sostiene que el hablante usa la CP con se (en lugar de la CP con ser) cuando no intenta describir el traspaso de energía ni el cambio en el paciente, sino destacar una figura contra un fondo en un proceso de intransitivización que elige el paciente como pivote (no el agente) que se acerca mucho al modelo de escenario (2005: 18-19) (como por ejemplo, en: En Roger Park se registraron cuatro tiroteos en calles distintas). Borzi comprueba sus hipótesis mediante el análisis de un corpus escrito compuesto de artículos periodísticos de la Ciudad de Buenos Aires.

\subsubsection{Los esquemas verbales de sentido pasivo}

Partiendo del MCI de bola de billar (cfr. ut supra) Borzi (2008) propone una clasificación de esquemas verbales, a partir de la cláusula transitiva prototípica

de la construcción pasiva (El muerto es honrado por los presentes - *lo es); 6. El significado pasivo es distinto del significado activo en lo nocional y en lo referencial (Juan financia el partido democrático es diferente de El partido democrático es financiado por Juan por la afectación del sujeto pasivo; a su vez: No vio a ninguna persona en el parque no se corresponde con Ninguna persona fue vista en el parque, que deriva de su grado de inaceptabilidad del distinto funcionamiento de la presuposición de existencia en el sujeto y en el objeto); 7. La reversibilidad no es siempre posible: * Libros son tenidos por Juan (Kovacci, 1990-1992)" (2005:37, n. xii)

9 Como por ejemplo, en: El sospechoso fue detenido a las 0.45 por efectivos de la comisaría $3^{a}$ y de la División Robos y Hurtos de la Policía Federal en Barracas. 
(se trata de esquemas verbales, puesto que el MCI se gramaticaliza en una cláusula, no sólo en el verbo). Desde esa zona central derivan los otros tipos de esquema, caracterizados por la pérdida o la acumulación de los distintos atributos de la cláusula prototípica, punto de partida de la organización de la categoría. Los esquemas muestran gradualidad en los atributos que los caracterizan y son no discretos (nos permitimos insistir aquí sobre el hecho de que el ECP sostiene una categorización gradual y no binaria, basada en la teoría de prototipos, según la cual dentro de cada categoría existe un 'mejor ejemplo' -el prototipo- y ejemplos no tan representativos de la categoría o marginales. Así, los atributos de una categoría pueden no estar todos presentes en sus miembros).

De esta caracterización de esquema verbal se desprende que los participantes de dichos esquemas (actantes) no son argumentos del verbo ni forman marco casual obligatorio (tal como se postula en los enfoques generativistas), sino que "cognitivamente lo preceden y es en la cláusula (es decir al combinarse con otro(s) actantes en un evento) donde se concretan las líneas interpretativas que corresponden a cada esquema" (Borzi, 2008: 1).

En la clasificación de esta autora, además de los esquemas transitivos prototípicos, tienen lugar esquemas donde se describen cambios de estado o proceso del actante involucrado. Estos son los esquemas que interesan a la hora de analizar el corpus del presente trabajo. Se trata de eventos de enfoque pasivo, que son pragmática, semántica y sintácticamente distintos de los eventos de enfoque activo y, en consecuencia, no se interpretan como derivados de la voz activa. En el evento pasivo hay dos actantes preexistentes, el paciente está en foco. Gráficamente, podríamos representarlo como un esquema de: +Energía con traslado, $(+\mathrm{Ag}),+\mathrm{Pac}$ (La puerta fue abierta por el edecán); +Energía sin traslado (cerca del MCI de escenario) (Se abrió la ventana). Aun más cerca del MCI de escenario, se encuentran los esquemas estáticos con el verbo estar, representados como: +Tema, -Energía, +Resultado (El hielo está roto). En los enfoques pasivos, se hace foco en el paciente, pero el esquema no es totalmente estático. Al igual que en el MCI de bola de billar, hay dos participantes preexistentes, percibimos cierto desplazamiento de energía, que produce un cambio de estado o resultado. Como se ha mencionado en el apartado anterior, para la CP con verbo ser interesa el proceso de cambio en el paciente; en la CP con pronombre se interesa destacar el paciente; mientras que en las cláusulas estáticas se hace hincapié en el resultado o simplemente se presenta una figura contra un fondo (La casa está rodeada de árboles).

Considerando los planteos anteriores, en el presente trabajo se entenderá la CP como una construcción en sí misma, es decir, como una predicación cuyos participantes tienen atributos propios. En este sentido, pensar la $\mathrm{CP}$ 
como construcción con atributos propios, y teniendo en cuenta el marco teórico general presentado en $\S 1$ ut supra, implica considerar como hipótesis válida que el agente introducido por la preposición de presenta características diferentes del agente introducido por la preposición por, tal como se demostrará en $\$ 4$ y ss., ut infra.

\subsection{El complemento agente}

Adentrándonos en el estudio del CAg, a continuación se presenta un breve estado de la cuestión sobre esta función sintáctica.

Es de destacar que no todas las gramáticas tradicionales describen el CAg. Entre las que lo mencionan, se encuentran la GRAE de 1931 y el Esbozo (1973), que dividen las oraciones pasivas en primeras y segundas, según aparezca o no el agente (en las primeras se explicita el CAg, mientras que en las segundas, no $)^{10}$. Además, concluyen que en el CAg se usa más por que de, debido a que consideran el agente como medio o instrumento de acción, y no como punto de origen de la misma (1931: §275).

Por su parte, Gili Gaya (1955) señala que el CAg puede aparecer con las preposiciones por o de, pero advierte: "En la lengua moderna ha desaparecido casi enteramente el empleo de la preposición de con este significado [el de agente de pasiva]" (\$193).

Lázaro Carreter (1980) remite a la división entre primeras y segundas, y señala: "El sujeto lógico [de la oración pasiva] puede presentarse como complemento agente, precedido de las preposiciones por o de: 1) La obra fue aplaudida por el público; 2) La obra fue precedida de una gran propaganda" (1980: 61).

Kovacci (1990-1992) también advierte que el agente se construye generalmente con la preposición por, aunque también acepta de: Fui consolado por un ruiseñor (R. Darío, Poesía II, 101); Ni amó a las mujeres ni fue amado de ellas (J. Ortega y Gasset, La deshumanización del arte, 95) (1990: §5.2.5).

De acuerdo con De Miguel (1992), el denominado complemento agente no siempre es agente: en oraciones como el prado está limitado por la valla; el valle está cruzado por el río; la población está rodeada por montañas, a menudo

10 "Las primeras constan de tres elementos esenciales: sujeto paciente, verbo en voz pasiva y complemento agente, que es siempre un nombre con la preposición por o de, v. gr.: las paces fueron firmadas por los plenipotenciarios; la felicidad es deseada de todos. (...) La oración segunda de pasiva se distingue de la primera en que no lleva el complemento agente, y por lo menos consta sólo de dos términos esenciales: sujeto paciente y verbo (...) la felicidad es deseada" (1931: §275). Por su parte, el Esbozo señala: "Cuando interesa el agente o productor de la acción, éste se añade al verbo como complemento introducido por medio de las preposiciones por o de; p. ej.: El actor fue aplaudidísimo por la concurrencia; Juan es respetado por sus vecinos; La noticia era ya conocida por (o de) todos" (1973: §3.5.2.). 
se ha llamado complemento agente al sintagma-por (en términos generativistas), aunque, desde un punto de vista semántico, no tiene por qué ser agente. En efecto, De Miguel señala que el sintagma-por puede ser una meta (los libros fueron recibidos por el periodista) o un tema, como en los ejemplos mencionados en los que una entidad ubicada en cierto lugar delimita ese lugar (el prado está limitado por la valla). La autora, además, añade que, desde el punto de vista sintáctico, está en discusión si el CAg es un adjunto o un argumento (discusión que no tiene sentido desde el ECP, ya que no hablamos de estructura argumental ni de marco casual sino de esquemas verbales con verbos y actantes).

Desde la GDLE, se destacan los trabajos de Mendikoetxea y de Bosque. Según Mendikoetxea (1999: §25.4.2.1.), en las oraciones activas Las montañas rodean la ciudad; La nieve cubre las montañas; Una valla cerca la finca: "el sujeto tiene la función semántica de tema no afectado (el elemento del cual se predica un lugar), mientras que el objeto tiene un significado locativo. En la perífrasis con estar, el elemento locativo es el sujeto sintáctico y el otro argumento verbal se expresa por medio de un sintagma preposicional introducido por de o por": La ciudad está rodeada de montañas; Las cumbres están cubiertas de nieve; La finca está cercada por una valla. Para esta autora, en estos ejemplos, las construcciones de montañas, de nieve y por una valla no serían CAg sino simplemente sintagmas preposicionales.

Bosque, por su parte, advierte que la gramática de los complementos agentes es más compleja en el caso de las $\mathrm{CP}$ con estar que en el de las que $\mathrm{CP}$ con ser. Sobre todo con verbos que el autor denomina transitivos estativos, como rodear, ocupar, sitiar, cercar, formar, habitar, constituir, integrar, y otros verbos que no describen necesariamente acciones, sino propiedades de las entidades de las que se predican (Bosque, 1999: §4.4.5.1.). Bosque señala que es en cierta forma paradójico que estos verbos de estado admitan los llamados complementos agente sin que en realidad tenga lugar ninguna acción, como en las oraciones: La ciudad está rodeada por murallas; Se trata de una sociedad deportiva que está formada por jóvenes de ambos sexos menores de 30 años; Madrid está habitado por cuatro millones de personas. A esa clase se añade la de los transitivos de afección (admirado por todos, respetado por sus compañeros, conocido por mucha gente, odiado por los que protegió), también estativos.

Para Bosque, los participios de los verbos transitivos construidos con complementos preposicionales agentivos mantienen o heredan el argumento externo del verbo. El que no exista intrepretación eventiva en estos participios es un rasgo esencial que los acerca a los adjetivos y los diferencia de los participios más puramente verbales, como los de las pasivas con ser. Como es de esperar, se denota acción (y por tanto agente) en las variantes agentivas de los 
verbos citados, lo que indirectamente produce la interpretación activa (y hasta la presencia de movimiento) que sugiere la oración En este momento la ciudad es rodeada por las tropas enemigas, frente a la situación estática que se describe en En este momento la ciudad está rodeada por las tropas enemigas. A partir de esta distinción, Bosque concluye que los complementos preposicionales citados no son argumentos agentes en las construcciones con estar sino complementos pseudoagentivos.

En cuanto a la preposición que puede introducir CAg, Bosque menciona las preposiciones por o de. La preposición por es la preposición no marcada, de uso más general en la lengua actual, según el autor. La preposición de, según Bosque, se emplea en la actualidad de forma mayoritaria en las construcciones estativas ${ }^{11}$.

Por último, la GRAE de 2009 menciona que el CAg de las pasivas reproduce el sujeto de las activas, pero en otra función sintáctica. Además, afirma que es polémica la cuestión de si estos complementos son argumentos o adjuntos: "A favor de la segunda opción se suele aducir que la sintaxis de la construcción pasiva los presenta como segmentos omisibles, ya que el hablante desea centrar la predicación en la aseveración que aporta sobre el sujeto paciente" (\$41.31), mientras que "a favor del complemento agente como argumento cabe señalar que muchos complementos agente no pueden omitirse ( $\mathrm{La}$ película fue interpretada por Jorge Negrete)" $(41.3 \tilde{n})^{12}$. Lo interesante de este comentario es que se apela a la Semántica para reconocer una función sintáctica, en lugar de valerse de pruebas formales. En cuanto a la alternancia de las preposiciones de y por en los CAg (como en conocido de todos/por todos; rodeada de montañas/por montañas), la GRAE postula que esta alternancia está relacionada con las propiedades léxicas de los participios, y no tanto con la naturaleza gramatical de las oraciones pasivas (\$27.8p). Sin embargo, esto no explica por qué por alterna con de en oraciones con el mismo verbo, como bien se demostrará en los análisis de los corpora recogidos para el presente trabajo.

11 Bosque clasifica el empleo de la preposición de en el CAg según el tipo de construcción estativa: a) predicados de afección: (aborrecido de todos, temido de la gente); b) predicados estativos que indican forma, posición y locación, como los citados del tipo de rodear, abrazar, sitiar, acompañar, seguir, preceder (vive rodeado de enemigos, iba precedido de un largo prólogo); c) en la lengua antigua, más raramente en la actual, los complementos con la preposición de incidían también sobre predicados de influencia como amenazar, amonestar, apercibir, apremiar, castigar, confundir, aconsejar, consolar, constreñir, todos los cuales se usan con estar y complemento agente con por en la actualidad (de Dios seas perdonado). (1999: §4.4.5.1.) 12 A este respecto, la GRAE agrega: "A ello se añade que la posible omisión de los complementos agente no constituye un criterio gramatical objetivo. Cabría pensar, en efecto, que el complemento agente puede omitirse en Esta conversación fue escuchada por Raimundita, pero no es menos cierto que lo que el autor quiere expresar en este texto es que la persona que escuchó cierta conversación fue Raimundita, en lugar de otra" (2009: 41.3ñ). 
En síntesis, ninguno de los teóricos mencionados caracteriza el CAg ni sintáctica ni semánticamente: i) no explican el motivo de su aparición; ii) no mencionan que no es posible la prueba de conmutación por pronombre para el reconocimiento de esta función sintáctica; iii) no describen semánticamente el actante (rol) en sí. Eventualmente, atienden a la preposición que lo introduce. Coinciden en que por es la de mayor frecuencia, evocan la ocasional presencia de de, aunque no se valida la afirmación con datos empíricos ${ }^{13}$.

Desde el ECP y afines, ciertos autores han estudiado más en profundidad la función de CAg. Así, Miñones y Sánchez (2000) describen los actantes partiendo de la CP. Analizan un corpus escrito ${ }^{14}$ y concluyen que los atributos del agente introducido por la preposición por son: [+voluntad] y [+humano], en contextos con pacientes altamente afectados de rasgos [+determinado], [+individual] y [-humano]. No contemplan en su estudio el CAg introducido por de.

Por su parte, Delbecque (2003) $)^{15}$ propone que la voz pasiva no se limita a la opción de focalizar el agente o rebajarlo, sino que también permite modular la configuración de los roles participativos de un modo incluso más sutil y variado que la voz activa. En este sentido, analiza la alternancia de las preposiciones por, de, con y en en el CAg. En cuanto a la preposición por, esta autora

13 De los estudios aquí reseñados, sólo los trabajos de De Miguel, Mendikoetxea y Bosque intentan una caracterización semántica del CAg. Advierten, por ejemplo, que no siempre se trata de agentes y que tienen un comportamiento distinto según se construyan con el verbo ser o estar. Sin embargo, ilustran sus afirmaciones con ejemplos ad hoc que no tienen realidad concreta en el uso de la lengua, como el varias veces citado ejemplo la población está rodeada por montañas, donde la concurrencia de rodear con la preposición por no suele darse en la lengua actual, como se refleja en el corpus analizado en el presente trabajo. El problema, insistimos, está en la falta de validación de las hipótesis con datos empíricos.

14 Las autoras analizaron la CP de frase verbal con ser en el discurso narrativo (tomando un libro de cuentos: Al Capitán de los duendes) y en el discurso histórico (tomando el texto La República perdida).

15 Esta autora distingue siete formas de predicado con significado pasivo: 1. Pasiva auxiliada, es decir, compuesta de un auxiliar seguido de un participio pasado: esto incluye los verbos ser y estar ("sus vidas fueron fracturadas y avasalladas por una orden que usted dio"); 2. Construcción participial adnominal ("los miembros de las juventudes comunistas, de manos empuñadas, rodeados de soldados con ametralladores, empezaron a cantar la Internacional"); 3. Pasivas reflejas ("No habiendo ocurrido ni lo uno ni lo otro, las soluciones democráticas deben derivarse de las proposiciones democráticas"); 4. Construcciones medias ("Europa puede beneficiarse de esta dinámica regional favorable"); 5 . Causativas reflejas, es decir, los casos en los que el clítico reflexivo se asocia con un auxiliar causativo seguido de un infinitivo ("Nosotros no podíamos (...) dejarnos llevar por nuestras emociones"); 6 . Predicación secundaria, con un participio que predica al complemento de objeto directo ("el Ejecutivo no posee una mayoría en el Senado y continuamente ve sus proyectos vetados por la oposición”) (2003: 6-8). 
plantea que suele denotar entidades animadas y colectivas. Dado que tienen por naturaleza un carácter delimitado, estas entidades están en una relación de discontinuidad con la entidad sujeto. "Condicionan" el perfil predicacional de manera tan sustancial que su impacto tiende a ser concebido en términos de "responsabilidad". Además, estos agentes aparecen típicamente en construcciones participiales y pasivas auxiliadas (2003: 10). En relación con la preposición de, Delbecque plantea que en general esta preposición introduce estados de cosas, eventos y estados de ánimo, es decir, entidades de contornos más bien inespecíficos, vagos, que se sitúan en una relación de continuidad con la entidad sujeto. En términos cuantitativos, los oblicuos con de presentan una primera concentración en construcciones participiales (el 41,4\%), y otra en construcciones medias (el 36,2\%), a expensas de las pasivas reflejas (el 1,7\%) (2003: 9).

Finalmente, Borzi (2004) analiza el CAg introducido por la preposición por en la CP de frase verbal con ser: el 'agente', aunque no es tópico, es conocido del contexto previo y, en consecuencia, se mantiene vigente. Así, cuando el hablante quiere mantener la cadena tópica y el agente es conocido o no interesa, el orden típico es (1) paciente + ser + participio; cuando el hablante quiere presentar al agente como información nueva opta por el orden (2) paciente + ser + participio + agente y, por último, cuando el hablante quiere presentar como información nueva al paciente opta por el orden (3) ser + participio + paciente, omitiendo la mención del agente. La aparición del CAg es dependiente de la distribución de la información y tiende a darse en contextos con paciente conocido en posición 1. Asimismo, Borzi no estudia los casos de CAg con de manifestados en su cuerpo de datos ${ }^{16}$.

Considerando todo lo anteriormente reseñado, en el presente trabajo, se entenderá la noción de complemento agente como la construcción que manifiesta al actante ${ }^{17}$ agente en una predicación con sentido pasivo. Se tomará el

16 "En cuanto al llamado Complemento Agente, como cabe interpretar como Agentes tanto construcciones introducidas por por (El representante fue recibido por los vecinos), como construcciones introducidas por de (esas declaraciones son conocidas de todos, los postulantes fueron elegidos de la convención radical de julio y agosto último, las autoridades argentinas fueron invitadas por el país anfitrión y de los Estados Unidos) como construcciones introducidas por con (los choques fueron disminuidos con el uso del semáforo/ Los administradores fueron autorizados con poderes especiales), así como construcciones con a causa de/como consecuencia de (la niña fue despedida a causa de un impacto) y con en (fue trasladado en helicóptero): trabajamos solamente con aquellos casos cuyo Complemento Agente estuviera introducido por la preposición por" (2000: 7).

17 Desde el ECP, la noción de actante (lo que para otras teorías serían argumentos, papeles temáticos o semánticos, o casos) se refiere a "entidades móviles, cambiantes en todos y en cada uno de sus atributos que se redefinen cada vez en cada mensaje al combinarse con cada verbo y con cada otro actante en un contexto determinado. Es decir, que así como no hay 
término agente en un sentido convencional, ya que se tiene en cuenta la escala de agentividad que surge de la caracterización de los esquemas verbales ya explicados. Partiendo de la cláusula transitiva prototípica, el actante agente presenta una escala desde el más prototípico hasta el más marginal: agente (Juan pinta la pared), experimentante (Juan piensa), benefactivo (Juan tiene frío), instrumental (La piedra rompió el vidrio), tema (El lápiz está sobre la mesa). Los subtipos de agente son las distintas formas en que este actante se configura en una predicación que es esencialmente relacional.

Esta concepción de CAg justifica que consideremos agentes sintagmas del tipo una veredita en casos como La casa está rodeada de una veredita. Sin embargo, se tiene en cuenta que no se trata de un agente prototípico, sino de un ejemplo no central para la categoría, ejemplos de los que nos ocupamos en este artículo.

\section{Problema(s) pendiente(s)}

De lo previamente expuesto se concluye que, por un lado, los autores que analizan la CP desde un enfoque formal no reconocen diferencias semánticas en los actantes involucrados. Sólo reconocen el cambio en las funciones sintácticas.

En contraste, los autores que analizan la CP desde enfoques funcionalistas o cognitivistas advierten que existen diferencias sustanciales entre los participantes de la cláusula activa y los de la cláusula pasiva (porque a cada estructura sintáctica le corresponde un significado específico).

Sin embargo, no hay acuerdo absoluto acerca de cuáles son los atributos más típicos de los participantes del evento pasivo. Además, no todos los autores describen los atributos, sino que presentan una explicación o caracterización general del fenómeno. Tampoco describen la (posible) diferencia sintáctico-semántica entre los CAg introducidos por diferentes preposiciones.

Atendiendo a estos problemas y con el objetivo de completar el estudio previo donde sólo se analizaba el CAg introducido por de (en corpora orales), en el presente trabajo se caracterizarán los atributos del agente introducido por la preposición por, en su contexto preferido de aparición, y se reforzará el estudio con un análisis de corpus escrito donde se relevará la presencia de CAg con ambas preposiciones.

verbos en tanto lexemas fijos con características fijas, tampoco hay actantes preexistentes de características fijas y obligatorias, tanto en la ausencia/presencia de esas características como en el grado de cada una de esas características. Es cada una de las combinatorias las que definen a cada uno de los elementos que componen el evento" (Borzi, 2008: 1). 


\section{Hipótesis}

\subsection{Antecedentes: el complemento agente introducido por la preposición de}

En un estudio previo (Funes, en prensa), se describió el CAg introducido por la preposición de en su contexto preferido de aparición, prestando especial atención a los atributos de los actantes, más allá del tipo de verbo de la cláusula donde ocurrían. Se comparó el agente introducido por de con los atributos del agente y del paciente de la cláusula transitiva prototípica.

Se denomina 'cláusula transitiva prototípica' (CTP) a aquélla que representa una cadena de acción, es decir, una experiencia del hablante acerca del mundo, según la cual un sujeto se relaciona con un objeto (por ejemplo: Juan rompió el vidrio). La CTP es una unidad semántico-pragmática que presenta una interacción, que tiene un movimiento de energía que parte del primer actante, se desplaza, cae en el último actante y provoca un cambio radical en él. Este traspaso de energía que provoca una modificación en el segundo actante es lo que las gramáticas han denominado transitividad. Sin embargo, para el ECP, la transitividad no es una característica del verbo sino del mensaje, de la cláusula.

Esto se refleja en el planteo de Hopper y Thompson (1980: 251-253), según el cual la transitividad es una propiedad global de la cláusula, que consiste en la transmisión efectiva de energía desde un agente hacia un paciente ${ }^{18}$.

Los autores caracterizan la cláusula transitiva prototípica de la siguiente manera: los participantes necesariamente son dos (sujeto agente y objeto paciente), ya que no puede haber transferencia de energía si no hay al menos dos participantes involucrados. La energía que se transmite proviene de una acción, por lo que debe haber kinesis (movimiento, evento dinámico, gramaticalizado en un verbo de acción). El agente reúne los siguientes atributos: [+humano], [+punto de partida], [+pivote $]^{19}$ [+ejecutor], [+iniciador], [+voluntad], en tanto que el cambio en el paciente provocado por la energía que le transmite el agente es total (es decir, hay alta afectación).

Otras características de la cláusula transitiva prototípica: aspecto télico, acción puntual (se perciben el inicio y el final de la acción de forma clara, por ejemplo: Juan pateó la pelota es más puntual que Juan llevó la pelota al armario), cláusula afirmativa y modo verbal indicativo.

Para estos autores, además, las cláusulas transitivas son las más frecuentes en el discurso, y eso deriva de la correlación entre la transitividad y la organi-

18 " [...] transitivity is [...] a global property of an entire clause, such that an activity is 'carried-over' or 'transferred' from an agent to a patient" (1980: 251).

19 El pivote de una estructura sintáctica es la frase nominal en torno a la que se organiza el enunciado (Foley y Van Valin, 1984: 111). 
zación del discurso en términos de cláusulas principales (foregrounded clauses) y cláusulas secundarias (backgrounded clauses): "The grammatical and semantic prominence of Transitivity is shown to derive from its characteristic discourse function: high Transitivity is correlated with foregrounding, and low Transitivity with backgrounding" (1980: 251).

Para corroborar esta hipótesis de que la transitividad se correspondía con el contenido principal del discurso, Hopper y Thompson analizaron un gran número de lenguas y comprobaron que sucedía lo mismo en todas: las cláusulas principales de un texto se corresponden con las cláusulas transitivas.

Dada la cláusula transitiva prototípica, hay diferentes grados de prominencia en la conceptualización de los participantes de un evento, lo cual se refleja a su vez en las estructuras gramaticales utilizadas como expresión. A partir de la construcción verbal básica (transitiva), podemos incrementar o disminuir el número de actantes o la prominencia de los participantes en el evento. Entre las estructuras que reducen la prominencia, se encuentra la CP. Desde una perspectiva cognitiva, toda diferencia sintáctica refleja un cambio semántico. En consecuencia, la CP tiene significado propio.

En contraste con los atributos de la cláusula transitiva prototípica, en las cláusulas donde aparece la preposición de introduciendo la función sintáctica de CAg, los actantes 'agente' y 'paciente' presentan generalmente atributos opuestos a los esperados en la cláusula activa. Así, el agente suele tener los atributos [-humano] y [-determinado], mientras que el grado de afectación del paciente es bajo o nulo. Además, el CAg introducido por de ocurre en esquemas de predicaciones con entidades estáticas preexistentes (Funes, en prensa: $\$ 5)^{20}$.

20 Véase a modo ilustrativo, un ejemplo de corpus oral que corrobora la hipótesis: "Enc. -Ahora usted me habló de-- población. ¿A qué le llama población? Inf. -Bueno-- mire- las estancias... eh... siempre... eh si ustedes hubieran ido al campo-- hubieran oído decir- que- - a las estancias - la gente de campo le llama 'las casas'. No le dice 'la casa', le dice 'las casas'. Enc. - ¿Por qué es eso? Inf. -Porque nunca es una casa sola. Por ejemplo, está... eh... nosotros tenemos la casa principal-- que en el campo le llaman el chalet-- la gente de campo, aunque no sea un chalet, como lo nuestro no es un chalet. Es una casa de así- de estilo- no diríamos de estilo español. Yo no sé- no podría decirle- es una casa de cincuenta años-- pero está sola rodeada de una veredita." (HCCBA, muestra XVI). En la descripción ofrecida, el hablante señala que la casa principal donde vive "está sola rodeada de una veredita". Vemos que el agente introducido por la preposición de reúne los atributos [-humano] y [-determinado]. La construcción es de verbo 'estar + atributo'. La interpretamos como pasiva porque podemos reconocer a los dos participantes del evento bola de billar: 'Una veredita rodea la casa'. Por último, se observa que el paciente se encuentra poco afectado (es un 'paciente locativo'), ya que el verbo rodear no implica cambio, es más bien una percepción estática: se recorta la figura casa contra un fondo donde se observa la veredita. 


\subsection{Hipótesis propuestas}

En corpora orales, el CAg introducido por la preposición por tiende a aparecer en contextos con pacientes altamente afectados y con agentes [+humanos] y [+determinados], en esquemas de predicaciones de eventos dinámicos (en contraste con el CAg introducido por de analizado en Funes, en prensa, tal como se describe en 3.1.).

En corpus escrito, se corroboraría la hipótesis sobre el CAg introducido por la preposición por (según la cual, por tiende a aparecer en contextos con pacientes altamente afectados y con agentes [+humanos] y [+determinados], en esquemas de predicaciones de eventos dinámicos), y la hipótesis sobre el CAg introducido por de (según la cual, la preposición de tiende a acompañar a agentes [-humanos] y [-determinados], en contexto de pacientes con baja afectación, y en esquemas de predicaciones con entidades estáticas).

\section{Metodología y corpus}

El marco teórico del presente trabajo supone una metodología basada en un análisis cuantitativo y cualitativo, ambos realizados sobre un cuerpo de datos auténtico.

Por análisis cualitativo se entiende la evaluación sistemática de los contextos en los que se produce el fenómeno estudiado. En estos contextos, se observó en primer lugar la presencia de los atributos [+/-humano] y [+/-determinado] para el participante agente introducido tanto por la preposición por como por de.

Por análisis cuantitativo se entiende contabilizar la presencia de CAg introducidos por la preposición por y aquéllos introducidos por de en los corpora recogidos. Dadas las hipótesis propuestas, se midieron los siguientes atributos en el actante 'agente': [+/-humano], [+/-determinado], y luego se extrajeron comparativamente los porcentajes. Se midió, además, el grado de afectación del paciente, teniendo en cuenta la jerarquía de subtipos propuesta por Borzi $(2004)^{21}$. Cabe aclarar que, en cuanto a la naturaleza léxica del verbo, se trabajó sin discriminar los subtipos.

21 Borzi registra los siguientes subtipos de pacientes, que presenta en una escala desde el que manifiesta menor afectación al que manifiesta mayor grado de afectación: paciente locativo: es aquel que se corresponde con un paciente que es la posición local o la extensión espacial que recibe la actividad descripta por el verbo (el comerciante fue abordado por los delincuentes); paciente objeto: es aquel que se corresponde con el objeto implicado en el proceso descripto por el verbo sin sufrir afectación (el féretro fue llevado al Cementerio de la Recoleta); paciente completante: es aquel que se corresponde con el objeto de un verbo 
Se relevó la presencia de CAg introducido por la preposición por en los siguientes corpora orales: 1) El habla culta de la Ciudad de Buenos Aires. Materiales para su estudio $(\mathrm{HCCBA})^{22}$, donde están registrados usos de la década de 1960; y 2) 31 entrevistas radiales (ER) ${ }^{23}$ grabadas y transcriptas entre abril de 2004 y marzo de 2005, recogidas por la Mag. Laura Miñones. Se tuvieron en cuenta sólo las entrevistas donde aparecían CAg introducidos por de.

Además, se relevó la presencia de CAg introducidos por por y por de en un corpus escrito de discurso periodístico compuesto por 21 noticias extraídas de los diarios argentinos Clarín y La Nación, recogidas entre los años 2009 y 2010.

\section{Análisis}

\subsection{Análisis de los corpora orales}

A continuación, se analizan cualitativamente dos ejemplos de CAg introducidos por la preposición por, con el propósito de observar cómo operan los atributos [+/-humano] y [+/-determinado] en un contexto auténtico de uso:

\subsubsection{Ejemplo de agente [-humano] y [-determinado] introducido por la preposición por}

En el ejemplo (1), se hace referencia a la baja de efectivos de la Policía Bonaerense que había ordenado el ministro de Justicia de esa época (año 2004), León Arslanian, luego de una ola de secuestros. Sin embargo, el Comisario

de acción y cuyo contenido está de un modo u otro implícito en la base (la ópera fue cantada por L.P.); paciente contenido de la percepción: es aquel que se corresponde con una frase nominal que manifiesta el contenido de la percepción, del pensamiento, de la idea, de la voluntad o sentimiento (esas palabras fueron dichas por el Ministro del Interior); paciente experimentante: es aquel que identifica al ser viviente afectado por algún proceso de tipo psicológico o de sensación (el fundador fue recordado en una sentida ceremonia); paciente benefactivo: es el caso que identifica el resultado de un proceso que desemboca en posesión (Paula Cahen fue nombrada Presidenta de Exxel); paciente/cambio de estado: este caso aparece con verbos no-psicológicos que indican cambio de estado (el delincuente fue asesinado a mansalva por la policía); paciente/factitivo: es el caso del objeto o ser que resulta de la acción identificada por el verbo (la receta fue extendida por el mismo profesional) (2004: §4.1). 22 El HCCBA está compuesta por 33 muestras que comprenden 21 horas y 20 minutos de grabación. Se distinguen en este cuerpo de datos una primera parte de entrevistas informales con grabador a la vista (entrevistas I-XVI); una segunda parte de elocuciones formales (muestra XVII-XX); una tercera parte de diálogos libres (muestras XXI-XXIX) y una cuarta parte de encuestas secretas (muestra XXX-XXXIII).

23 Estas entrevistas fueron realizadas en el marco de programas radiales informativos de actualidad de la ciudad de Buenos Aires, y en ellas intervienen, en todos los casos, más de dos participantes. Los hablantes de estas entrevistas son argentinos, hablantes nativos del español, poseen formación secundaria completa o superior; 31 de estos hablantes son de sexo masculino y 15 de sexo femenino. Cada entrevista tiene una duración de entre 15 y 20 minutos. 
Mayor Cabrera no había acatado las órdenes y todavía permanecían policías corruptos en ejercicio de sus funciones. En relación con este incidente, Arslanian observa:

(1) A: Respecto del Comisario Mayor Cabrera, es...eh....obvio que como Jefe de la Departamental tenía la obligación de controlar...eh...el desempeño de su personal, el tomar las medidas disciplinarias, este, correctivas y hasta...este... represivas respecto de quienes eh venían incumpliendo severamente con sus obligaciones o veí, o veían, o se veían involucrados en delitos o en corrupción, medidas que no tomó...este... no llevó a cabo, pese a que la Departamental eh exhibe un elevado grado, o ha estado asolada, por delitos de distinta naturaleza, entre ellos, secuestro extorsivo (ER 5, León Arslanian, audio grabado, Un día por delante).

En el comentario ofrecido, Arslanian señala que la Departamental "ha estado asolada por delitos de distinta naturaleza, entre ellos, secuestro extorsivo". En primer lugar, se observa que el agente introducido por la preposición por reúne los atributos [-humano] y [-determinado]. La construcción sintáctica es de verbo 'estar + atributo'; puede ser interpretada como pasiva porque puede reconocerse a los dos participantes del evento: 'Delitos asuelan la Departamental'. En segundo lugar, se observa que el paciente se encuentra altamente afectado (es un 'paciente cambio de estado'), a esto contribuyen la semántica del verbo asolar y de los agentes delitos y secuestro extorsivo. El evento se percibe, entonces, como altamente dinámico. A pesar de ser agentes [-humanos] y [determinados], la elección de la preposición por estaría motivada por la característica dinámica del evento y por la alta afectación del paciente.

6.1.2. Ejemplo de agente [+humano] y [+determinado] introducido por la preposición por

En el ejemplo (2), en referencia a un secuestro ocurrido en octubre de 2004, un periodista comenta:

(2) S: Después vamos a hablar del abogado que fue secuestrado en ese grupo de personas que se llevaron a San Martin y que afortunadamente fueron liberados después. Había dos chicos, la novia de uno de ellos de apellido Neumann que dice que fue muy maltratada por los secuestradores, un abogado y su amigo...eh... El abogado, ya lo dijimos en los títulos, dijo: "Los jueces no tienen cojones, yo no voy - eh textual lo que dijo el abogado- no voy a declarar. Aunque me acuerde la cara, aunque sepa el nombre y tenga el documento de identidad del que me secuestró, no digo nada porque van a tomar represalias contra mi familia" (ER 6, Franco Salomone, Un día por delante).

En el ejemplo, el periodista advierte que la novia de uno de los damnificados, según su propio testimonio, "fue muy maltratada por los secuestradores, 
un abogado y su amigo". En este caso, el agente introducido por la preposición por (en realidad, son tres) reúne los atributos [+humano] y [+determinado]. La construcción es pasiva de frase verbal con ser. El paciente se encuentra altamente afectado (es un 'paciente cambio de estado'), a esto contribuye la semántica del verbo maltratar, mientras que el evento es dinámico. La elección de la preposición por, por lo tanto, estaría motivada por la conjunción de todos los factores mencionados: agente [+humano] y [+determinado], paciente altamente afectado y evento dinámico.

\subsubsection{Resultados y discusión}

Tomando los dos ejemplos contrapuestos, se verá si la tendencia sigue a (1) o a (2). Los resultados del análisis indican que sobre un total de 43 ocurrencias de CAg con por (29 casos en el HCCBA y 14 casos en las ER), 17 $(39,53 \%)$ corresponden a agentes con el atributo [-humano] y $10(71,43 \%)$ a agentes [-determinados], mientras que $26(60,46 \%)$ corresponden a agentes [+humanos] y $33(76,74 \%)$ a agentes [+determinados]. Véase la Tabla I para la comparación con los CAg introducidos por de analizados en los mismos corpora orales, en Funes (en prensa: \$5).

\begin{tabular}{l|c|c|c|c}
\hline \multirow{2}{*}{ Prep. introductora del CAg } & \multicolumn{4}{|c}{ Atributos } \\
\cline { 2 - 5 } & {$[$-humano] } & [+humano] & [-determinado] & [+determinado] \\
\hline DE & $10(71,43 \%)$ & $4(28,57 \%)$ & $10(71,43 \%)$ & $4(28,57 \%)$ \\
\hline POR & $17(39,53 \%)$ & $26(60,47 \%)$ & $10(23,26 \%)$ & $33(76,74 \%)$ \\
\hline
\end{tabular}

Tabla I. Atributos del agente en el CAg según la preposición introductora (corpus oral)

En cuanto al grado de afectación del paciente, se observa que hay 9 casos de baja afectación (20,93\%), que se reparten entre los subtipos 'paciente objeto' y 'paciente locativo', mientras que el resto de los pacientes presenta media o alta afectación (34 casos: 79,07\%) y se corresponden con el subtipo 'paciente cambio de estado'.

Finalmente, con respecto a la percepción del evento, se observan 30 casos de eventos dinámicos $(69,77 \%)$ y 13 casos de esquemas estáticos $(30,23 \%)$, distribuidos en distintas estructuras sintácticas, como puede verse en la Tabla II, donde además se ofrece la comparación con los CAg introducidos por de: 


\begin{tabular}{l|c|c}
\hline Tipo de estructura sintáctica & CAg introducido por 'de* & CAg introducido por 'por' \\
\hline Pasiva de frase verbal con 'ser' & $0(0 \%)$ & $8(18,60 \%)$ \\
\hline Pasiva con 'se' & $3(21,43 \%)$ & $6(13,95 \%)$ \\
\hline Pasiva con 'estar' & $7(50 \%)$ & $10(23,26 \%)$ \\
\hline Predicación no finita & $4(28,57 \%)$ & $19(44,19 \%)$ \\
\hline
\end{tabular}

* Los resultados provienen del estudio previo ya citado (Funes, en prensa: §5)

Tabla II. Tipo de estructura sintáctica según la preposición introductora del CAg (corpus oral)

Los corpora orales revelan, entonces, que, para el CAg introducido por la preposición por, hay un predominio de agentes con los rasgos [+humano] y [+determinado] y de pacientes con media o alta afectación (preeminencia de subtipo 'paciente cambio de estado'), en contexto de eventos dinámicos, y en estructuras sintácticas de predicación no finita (19 casos/44,19\%).

En la Tabla III puede observarse un resumen de la alternancia entre CAg introducidos por de y aquéllos introducidos por la preposición por en discurso oral:

\begin{tabular}{l|c|c}
\hline Parámetro & CAg introducido por 'de** & CAg introducido por 'por' \\
\hline Tipo de agente & [-humano] & {$[+$ humano] } \\
\hline Tipo de evento/esquema & Estático & Dinámico \\
\hline Grado de afectación del paciente & Bajo & Medio o alto \\
\hline Tipo de estructura sintáctica & Pasiva con 'estar' & Predicación no finita \\
\hline
\end{tabular}

* Para un análisis más profundo del CAg introducido por 'de' en corpus oral, véase Funes (en prensa)

Tabla III. Características del CAg según la preposición introductora (en corpus oral)

\subsection{Análisis del corpus escrito}

A continuación, se examinan cualitativamente dos ejemplos de cada preposición para ver cómo operan los atributos [+/-humano] y [+/-determinado] en contextos auténticos de uso:

\subsubsection{Ejemplo de agente [-humano] y [-determinado] introducido por por}

En el ejemplo (3), se hace referencia a una inundación muy fuerte ocurrida en Buenos Aires en el mes de febrero de 2010 a causa de lluvias intensas (se habían inundado los barrios de Palermo, Belgrano, Flores y Villa Crespo): 
(3) La lluvia retornó hoy al área metropolitana, seriamente afectada el lunes pasado por un temporal que hizo colapsar el sistema de drenajes, e hizo bajar en pocas horas 10 grados la sensación térmica, que llegó a superar los 38 grados, aunque también provocaba caos de tránsito por la escasa visibilidad. " La ciudad colapsó por el diluvio y se prevé que lloverá hasta mañana", La Nación, viernes 19 de febrero de 2010).

En la nota, el periodista señala que la ciudad había sido afectada "por un temporal", con tal intensidad que provocó un colapso en el sistema de drenajes y en el tránsito. En primer lugar, se observa que el agente introducido por la preposición por reúne los atributos [-humano] y [-determinado]. La construcción sintáctica es de predicación no finita. En segundo lugar, se observa que el paciente se encuentra altamente afectado (es un 'paciente cambio de estado'), a esto contribuyen la semántica del verbo afectar, del agente temporal y del adverbio seriamente. El evento se percibe, entonces, como altamente dinámico: un temporal afectó seriamente el área metropolitana, ocasionando colapso y caos. La elección de la preposición por estaría motivada por la característica fuertemente dinámica del evento y por la consecuente alta afectación del paciente.

\subsubsection{Ejemplo de agente [+humano] y [+determinado] introducido por por}

En el ejemplo (4), se alude a un episodio policial:

(4) Un taxista fue herido de un corte en el cuello por tres chicos que lo asaltaron en la ciudad de La Plata, lo que motivó una importante protesta de choferes que cortaron el acceso en la localidad de Berisso en reclamo de seguridad. Fuentes policiales confirmaron esta mañana que por el hecho fue demorado un adolescente de 16 años en Ensenada, al que le secuestraron un cuchillo ("Protesta de taxistas en Berisso tras el ataque a un chofer en un intento de robo", Clarín, viernes 19 de febrero de 2010).

El periodista describe que un taxista fue herido por tres asaltantes. En este caso, el agente (en realidad, son tres) introducido por la preposición por reúne los atributos [+humano] y [+determinado]. La construcción es pasiva de frase verbal con ser. Por último, se observa que el paciente se encuentra altamente afectado (es un 'paciente cambio de estado'), a esto contribuye la semántica del verbo herir. El evento es dinámico. La elección de la preposición por, por lo tanto, estaría motivada por todos los factores mencionados: agente [+humano] y [+determinado], paciente altamente afectado y evento dinámico.

\subsubsection{Ejemplo de agente [+humano] y [+determinado] introducido por de}

En (5), en un artículo sobre Miles Davis, se describe lo siguiente: 
(5) Davis, tan genial intérprete como compositor, supo siempre buscar el mejor cast, supo siempre encontrar y rodearse de los mejores compañeros de faena. Con todos ellos había colaborado con anterioridad, pero el simple listado de Julian Cannonball Adderley (saxo alto), John Coltrane (saxo tenor), Bill Evans (piano), Paul Chambers (contrabajo) y Jimmy Cobb (batería) ya quita el sueño ("Miles Davis alumbró hace medio siglo el nuevo y mejor jazz", Revista Ñ, 17 de agosto de 2009).

En el artículo, el periodista señala que Miles Davis siempre había sabido "rodearse de los mejores compañeros de faena" (a continuación, detalla quiénes son esos compañeros: Adderley, Coltrane, etc.). En este caso, el agente introducido por la preposición de reúne los atributos [+humano] y [+determinado]. La construcción es de pasiva con se no finita. El paciente se encuentra poco afectado (es un paciente locativo figurado) ya que el verbo rodear no implica transformación de la entidad afectada (cfr. con herir; afectar, de los ejemplos con por), es más bien una percepción estática, como si fuese un lugar, una entidad quieta, a la que se le acercan otras entidades. A pesar del agente [+humano], se entiende la elección de la preposición de por el resto de los elementos presentes: esquema estático, y paciente poco afectado.

\subsubsection{Ejemplo de agente [-humano] y [-determinado] introducido por de}

En (6) se observa un ejemplo dentro de un titular:

(6) En plena primavera, Bariloche amaneció cubierta de nieve

El fenómeno, que se inició durante la madrugada, generó una leve acumulación de nieve en las zonas céntricas y de hasta diez centímetros en los barrios altos. La temperatura mínima que se registró fue de 6 décimas de grado bajo cero y la sensación térmica de -8,2 grados (Clarín, viernes 23 de octubre de 2009).

En el ejemplo, vemos que el agente introducido por la preposición de reúne los atributos [-humano] y [-determinado]. La construcción es una predicación no finita. Por último, se observa que el paciente se encuentra medianamente afectado (es un paciente locativo), ya que en Bariloche suele nevar, y la característica que se destaca es que ocurrió en primavera. A pesar de la afectación del paciente, la percepción del esquema verbal es estático: se recorta una figura sobre un fondo, en este caso, un paisaje, la ciudad nevada. La elección de la preposición de estaría motivada por la naturaleza estática del esquema, que se percibe como resultado antes que como proceso: la nieve cubrió la ciudad de Bariloche y como resultado tenemos un paisaje nevado.

Confróntese con un ejemplo del corpus del mismo verbo y tipo de agente, pero diferente preposición:

En el ejemplo (6'), se hace referencia a una inundación muy extendida, provocada por lluvias intensas, en Buenos Aires, en febrero de 2010: 
(6') Entre otras de las imágenes que se repitieron como una reminiscencia del colapso del lunes, se pudo ver por las calles bolsas de basura flotando y autos prácticamente cubiertos por el agua, aún los de algunos que habían llegado a subirlos a la vereda. En la esquina de Humboldt y Santa Fe, un gomón ayudó a cruzar a algunas personas, mientras que otras iban de un lado a otro tomadas de una soga y otras más eran llevadas en andas por personal de Defensa Civil "Inundaciones, cortes de luz y caos: otra vez, la lluvia colapsó la Ciudad", Clarín, sábado 20 de febrero de 2010).

En la noticia, el periodista señala que en las calles de la ciudad se podían ver bolsas de basura y autos "cubiertos por el agua". En primer lugar, se observa que el agente introducido por la preposición por reúne los atributos [humano] y [+determinado]; la construcción sintáctica es de predicación no finita. En segundo lugar, se observa que el paciente se encuentra altamente afectado (es un 'paciente cambio de estado'), a esto contribuyen la semántica del verbo cubrir y la asociación semántica entre el agente agua y el tema de la nota: 'inundación'. El evento se percibe, entonces, como altamente dinámico: el agua cubrió por completo la ciudad debido a una fuerte inundación. A pesar de ser un agente [-humano], la elección de la preposición por estaría motivada por la característica dinámica del evento y por la alta afectación del paciente.

\subsubsection{Resultados y discusión}

Tomando los pares de ejemplos contrapuestos, se verá cuál es la tendencia que sigue el corpus escrito. Véase la Tabla IV para los resultados sobre los atributos del agente según la preposición introductora. Los porcentajes de todos los parámetros se midieron sobre un total de 35 casos de CAg introducido por por y un total de 13 casos de CAg introducido por de.

\begin{tabular}{l|c|c|c|c}
\hline \multirow{2}{*}{ Prep. introductora del CAg } & \multicolumn{4}{|c}{ Atributos } \\
\cline { 2 - 5 } & [-humano] & [+humano] & [-determinado] & [+determinado] \\
\hline DE & $8(61,54 \%)$ & $5(38,46 \%)$ & $9(69,23 \%)$ & $4(30,77 \%)$ \\
\hline POR & $14(40 \%)$ & $21(60 \%)$ & $9(25,71 \%)$ & $26(74,29 \%)$ \\
\hline
\end{tabular}

Tabla IV. Atributos del agente en el CAg según la preposición introductora (corpus escrito)

En cuanto al grado de afectación del sujeto paciente, se observa que en los CAg introducidos por por hay 31 casos de media o alta afectación (42,86\%), repartidos en los subtipos 'paciente cambio de estado' y 'paciente factitivo'; y tan sólo 4 casos de baja afectación $(11,43 \%)$, correspondientes a los subtipos 'paciente objeto' y 'paciente benefactivo'. Por su parte, en los CAg introducidos por de hay 11 casos de baja afectación $(84,62 \%)$, contra sólo dos casos de alta afectación (15,38\%). 
Con respecto a la percepción del evento, para el CAg introducido por por hay un $100 \%$ de casos de eventos dinámicos (35 ocurrencias), mientras que para el CAg introducido por de, el 100\% de los casos corresponde a esquemas estáticos (13 ocurrencias).

Por último, véase la Tabla V, donde se observa la alternancia de/por de los CAg según el tipo de estructura sintáctica donde ocurren.

\begin{tabular}{l|c|c}
\hline Tipo de estructura sintáctica & CAg introducido por 'de' & CAg introducido por 'por' \\
\hline Pasiva de frase verbal con 'ser' & $1(7,69 \%)$ & $18(51,43 \%)$ \\
\hline Pasiva con 'se' & $3(23,08 \%)$ & $1(2,86 \%)$ \\
\hline Pasiva con 'estar' & $2(15,38 \%)$ & $1(2,86 \%)$ \\
\hline Predicación no finita & $7(53,85 \%)$ & $15(42,86 \%)$ \\
\hline
\end{tabular}

Tabla V. Tipo de estructura sintáctica según la preposición introductora del CAg (corpus escrito)

El corpus escrito revela, entonces, que para el CAg encabezado por la preposición por hay un predominio de agentes [+humanos] y [+determinados], pacientes altamente afectados, en eventos dinámicos de CP de frase verbal con ser; mientras que para el CAg introducido por de hay un predominio de agentes [humanos] y [-determinados], en esquemas estáticos de predicaciones no finitas.

En la Tabla VI puede observarse una recapitulación de las condiciones de la alternancia entre CAg introducidos por de y CAg introducidos por la preposición por en corpus escrito:

\begin{tabular}{l|c|c}
\hline Parámetro & CAg introducido por 'de' & CAg introducido por 'por' \\
\hline Tipo de agente & [-humano] & {$[+$ humano] } \\
\hline Tipo de evento/esquema & Estático & Dinámico \\
\hline Grado de afectación del paciente & Bajo & Medio o alto \\
\hline Tipo de estructura sintáctica & Predicación no finita & Pasiva con 'ser' \\
\hline
\end{tabular}

Tabla VI. Características del CAg según la preposición introductora (en corpus escrito)

\section{Conclusiones}

Recapitulando, en corpus oral hay un uso marginal (baja frecuencia) de las CP en general. Dentro de estas construcciones, hay mayor frecuencia de aparición de CAg introducidos por la preposición por (43 casos de por contra 14 de de en un corpus de 20 entrevistas orales), tal como se señalaba en varias 
Gramáticas. En contraste con el CAg introducido por de, cuando el hablante elige la preposición por para introducir un $\mathrm{CAg}$, lo hace para acompañar agentes con los atributos [+humano] y [+determinado], en contexto de pacientes con media o alta afectación, y en esquemas de predicaciones dinámicas (manifestadas en su mayoría en predicaciones no finitas).

En corpus escrito, hay mayor frecuencia de CP, en relación con los corpora orales analizados en Funes (en prensa) ${ }^{24}$. Dentro de las CP, hay mayor frecuencia de aparición de CAg introducidos por por, en contraste con los introducidos por de. Cuando el hablante elige la preposición por para introducir un CAg, lo hace para acompañar agentes con los atributos [+humano] y [+determinado], en contexto de sujetos pacientes con media o alta afectación, y en esquemas de predicaciones dinámicas (manifestadas en CP de frase verbal con ser, en su mayoría). En contraste, cuando el hablante elige la preposición de para introducir un CAg, lo hace para acompañar agentes con el atributo [-humano] y [determinado], en contextos de sujetos pacientes con baja afectación, y en esquemas de predicaciones con entidades estáticas (manifestadas mayormente por predicaciones no finitas).

Las conclusiones extraídas del análisis en corpus escrito corroboran los resultados derivados del análisis en corpora orales (a excepción del tipo de estructura sintáctica predominante para ambas preposiciones).

\section{Referencias bibliográficas}

Alarcos Llorach, E. (1966): "Pasividad y atribución en español", Estudios de gramática funcional del español. Madrid, Gredos, págs. 163-171.

Alcina Franch, J. y J. M. Blecua: (1975): Gramática española. Barcelona, Ariel.

Alonso, A. y P. Henríquez Ureña (1938-1939): Gramática castellana. (2 vols.) Buenos Aires, Losada.

Bello, A. (1980 [1847]): Gramática de la lengua castellana. Madrid, EDAF.

Borzi, C. (2000): "La posición del Paciente en la Emisión de Voz pasiva de Frase Verbal de ser + participio". En Gramática, Discurso y Sociedad (Actas de las I Jornadas de Lingüistica "Beatriz Lavandera"), Universidad Nacional de Mar del Plata 24 al 27 de marzo de 1999, págs. 47-57.

Borzi, C. e H. Albano (2000): "Lineamientos generales del proyecto: 'Teoría prototípica y gramática aplicada a la enseñanza del español: la voz pasiva"' (UBACyTJO JF03). En Gramática, Discurso y Sociedad (Actas de las I Jornadas de Lingüística "Beatriz Lavandera"), Universidad Nacional de Mar del Plata, 24 al 27 de marzo de 1999, págs. 57-65.

24 Cabe aclarar que ningún autor reseñado ha trabajado con corpora orales dentro del ECP. Los análisis siempre fueron efectuados sobre la base de corpora escritos (en general, discurso periodístico y literario). 
Borzi, C. (2004): "La pasiva de ser + participio y los actantes en competencia", Signo ES Seña, 13, págs. 67-88.

Borzi, C. (2005): "La Función Sintáctica 'Sujeto Pasivo", Anales del Instituto de Lingüística (Universidad Nacional de Cuyo), XXIV-XXVI, págs.7-40.

Borzi, C. (2006): "Pasivas con 'se' y concordancia Sujeto/Verbo". En Actas del VII Congreso Nacional de Hispanistas, 19 al 22 de mayo de 2004, págs. 289-297.

Borzi, C. (2008): "Concepción de eventos y esquemas verbales". Ponencia Congreso Internacional: Debates Actuales. Las teorías críticas de la Literatura y la Lingüística, Depto.de Letras, FyL UBA, Buenos Aires, agosto de 2008.

Bosque, I. (1999): "El Sintagma Adjetival. Modificadores y complementos del adjetivo. Adjetivo y participio." En Bosque, I. y V. Demonte (dirs.): Gramática Descriptiva de la Lengua Española. Vol. 1. Madrid, Espasa Calpe, págs. 217-310.

Bosque, I. y V. Demonte (dirs.) (1999): Gramática Descriptiva de la Lengua Española. Madrid, Espasa-Calpe.

Brucart, J. M. (1990): "Pasividad y atribución en español: un análisis generativo". En Demonte, V. y B. Garza Cuarón (eds.): Estudios de lingüística de España y México. México D.F., UNAM, El Colegio de México, págs. 179-208.

Delbecque, N. (2003): "La variable expresión del agente en las construcciones pasivas", Nueva Revista de Filología Hispánica, LI, 2, págs. 373-416.

Foley, W. y R. Van Valin (1984): Functional Syntax and Universal Grammar. Cambridge, Cambridge University Press.

Funes, M. S. (en prensa): "El complemento agente introducido por la preposición 'de". En Actas del Primer Simposio de la Asociación Argentina de Lingüística Cognitiva (AALCO), 12 y 13 de noviembre de 2009. Mendoza, Universidad Nacional de Cuyo. Gili Gaya, S. (1955): Curso Superior de Sintaxis Española. Barcelona, Spes.

Givón, T. (1979): On understanding grammar. New York, Academic Press.

Hopper, P. y S. Thompson (1980): "Transitivity in Grammar and Discourse", Language, Vol. 56, 2, págs. 251-299.

Hopper, P. (1988): "Emergent Grammar and the A Priori Grammar Postulate". En Tannen, D. (ed.): Linguistics in Context: Connective Observation and Understanding. Ablex: Norwood, págs. 117-134.

Kovacci, O. (1990-1992): El Comentario gramatical I y II. Madrid, Arco Libros.

Lakoff, G. (1987): Women, fire and dangerous things. Chicago, University Press.

Langacker, R. (1987): Foundations of Cognitive Grammar. Theoretical Prerequisites. Vol. 1. Stanford, Stanford University Press.

Langacker, R. (1991): Foundations of Cognitive Grammar. Descriptive Applications. Vol. 2. Stanford, Stanford University Press.

Lázaro Carreter, F. (1980): "Sobre la pasiva en español”, Estudios de lingüística. Barcelona, Crítica, págs. 61-72.

Lenz, R. (1935): La oración y sus partes. Madrid, Nueva Revista de Filología Española. Mendikoetxea, A. (1999): "Construcciones inacusativas y pasivas". En Bosque, I. y V. Demonte (dirs.): Gramática Descriptiva de la Lengua Española. Vol. 2. Madrid, Espasa Calpe, págs. 1575-1630. 
Miguel, E. de (1992): El aspecto en la sintaxis del español; perfectividad e impersonalidad. Madrid, Ediciones de la Universidad Autónoma de Madrid.

Miñones, L. y K. Sánchez (2000): “Condiciones de aparición de la VpdeFV con 'ser' en el español escrito". En Gramática, Discurso y Sociedad (Actas de las I Jornadas de Lingüística "Beatriz Lavandera"), Universidad Nacional de Mar del Plata, 24 al 27 de marzo de 1999, págs. 1-12.

Real Academia Española. (1931): Gramática de la lengua española. Madrid, Espasa Calpe. Real Academia Española. (1973): Esbozo para una nueva gramática de la lengua española. Madrid, Espasa Calpe.

Real Academia Española. (2009): Nueva Gramática de la Lengua Española. Madrid, Espasa. Rosetti, M. M. de. (1969a). "La frase verbal pasiva en el sistema español". En Barrenechea, A. M. y M. M. de Rosetti: Estudios de gramática estructural. Buenos Aires, Paidós, págs. 71-90.

Rosetti, M. M. de. (1969b). "La llamada 'pasiva con se' en el sistema español". En Barrenechea, A. M. y M. M. de Rosetti: Estudios de gramática estructural. Buenos Aires, Paidós, págs. 91-99.

Seco, M. (1999). Gramática esencial de la lengua española. Madrid, Espasa.

Shibatani, M. (1985). "Passives and Related Constructions: A Prototype Analysis", Language, Vol. 61, 4, págs. 821-848. 